\title{
Performance Evaluation of Radar Range- Bearing Centroid Processing Using Time Series Analysis
}

\author{
Nguyen VAN LOI ${ }^{1}$, Tran QUOC TUAN, Tran TRUNG KIEN, \\ Tran VAN TRUONG, Tran VU HOP \\ ${ }^{a}$ Radar Center, Viettel High Technology Industries Corporation, Hanoi, Vietnam
}

\begin{abstract}
The paper deals with a problem of performance evaluation of the rangebearing centroid processing for a surveillance radar. First, we review several techniques for centroid processing that are Moving window estimator, Beam shape centroid estimator, Center of mass correlation and Recursive least-squares centroid estimator. Then we point out that the range root-mean-square error (RMSE) and bearing RMSE are not sufficient for performance evaluation of the range-bearing centroid processing. Further, a new parameter using time series analysis for evaluation of structural stability of the centroid processing is proposed. As an illustration, a test with data from an X-band coastal surveillance radar is given.
\end{abstract}

Keywords. Radar measurement, plot extractor, centroid processing, time series analysis

\section{Introduction}

Radar [1] is a system that uses the radio waves to detect the objects of various types such as aircraft, spacecraft, guided missiles, ships, motor vehicles, weather formations and terrain. A radar system consists of a transmitter producing electromagnetic waves in the radio or microwaves domain, a transmitting antenna, a receiving antenna (for a pulse radar the same antenna is used for transmitting and receiving) and a receiver and processor (see Figure 1).

\footnotetext{
${ }^{1}$ Corresponding Author. E-mail: loinv12@viettel.com.vn
} 


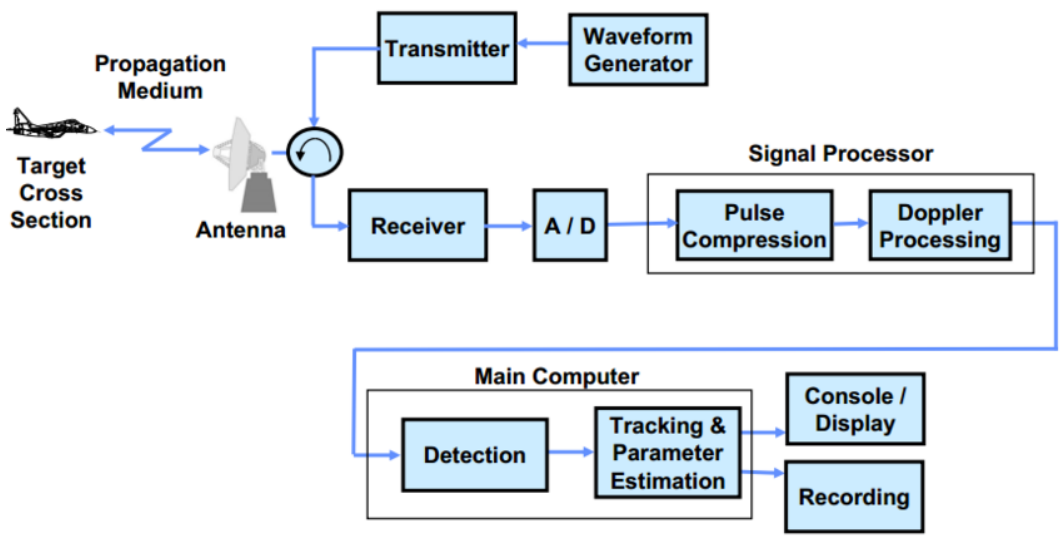

Figure 1. Radar block diagram [2]

The block "Detection" in Figure 1 contains the "Plot detector" and the "Plot extractor" modules (see Figure 2).

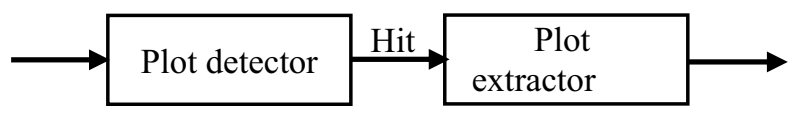

Figure 2. "Detection" block [3]

When the antenna scans across any target, there may be threshold crossings at the "Plot detector" in several inter pulse periods, and in several adjacent range and azimuth resolution cells. The output of "Plot detector" is called detected hits. The "Plot extractor" (or range-bearing centroid processing, centroid processing) takes the output of the signal processor of a radar system. Its function is to correlate all threshold crossings (all detected hits), grouping together all those which appear to come from the same target and to estimate range-bearing centroid for generating plot reports such as range, azimuth, power and radial velocity information on all validated targets. Then, the plot reports are used as input for "Tracking and Parameter estimation" block (see Figure 1) to perform object's trajectories which are displayed on the radar monitor. The more accurate plot reports of range-bearing centroid processing are, the better performance of a radar system is.

The present paper deals with the problem of performance evaluation of the "Plot extractor". Since the "Plot extractor" has an important role for the performance of a radar system, there are a lot of numbers in the literature concerning the problem of range-bearing centroid processing (see [4-8,10-12]). However, for performance evaluation there is only an approach mentioned in the literature which is based on the range and azimuth (bearing) RMSEs. As illustration in Section 2 (see Figure 7) the range and bearing RMSEs are not sufficient for evaluation of centroid processing. In fact, the target plot reports over scans form a time series and any break-point (changepoint) will strongly influence to the "Tracking and Parameter estimation" block. But the range and bearing RMSEs do not give us information about the existence of change-points in a time series. In this paper, a new parameter using time series analysis for evaluation of structural stability of range-bearing centroid processing is introduced.

The paper is organized in the following way. In the next section, we recall the common approaches for range-bearing centroid processing and its evaluation. The main contribution of the paper is presented in section 3. Last section gives out the conclusion and future works. 


\section{Related works}

The "Moving window estimator" is one of the earliest methods of correlating hits to form plot reports (see [4,5]). For each pulse repetition time (PRT), the detected hits are stored as " 1 "s in the range-cell dimension (see Figure 3 ). At every range cell, a window in the time-cell dimension is applied to count the number of " 1 " that are present in the window. The size of the window equals the number of reflected pulses when the radar antenna scans across a target. The target azimuth is estimated by (see $[4,6])$ :

$$
\hat{B}(k)=\frac{B\left(k_{1}\right)+B\left(k_{2}\right)}{2}
$$

where $B\left(k_{1}\right)$ and $B\left(k_{2}\right)$ are the beginning and end azimuth angles of the targets.

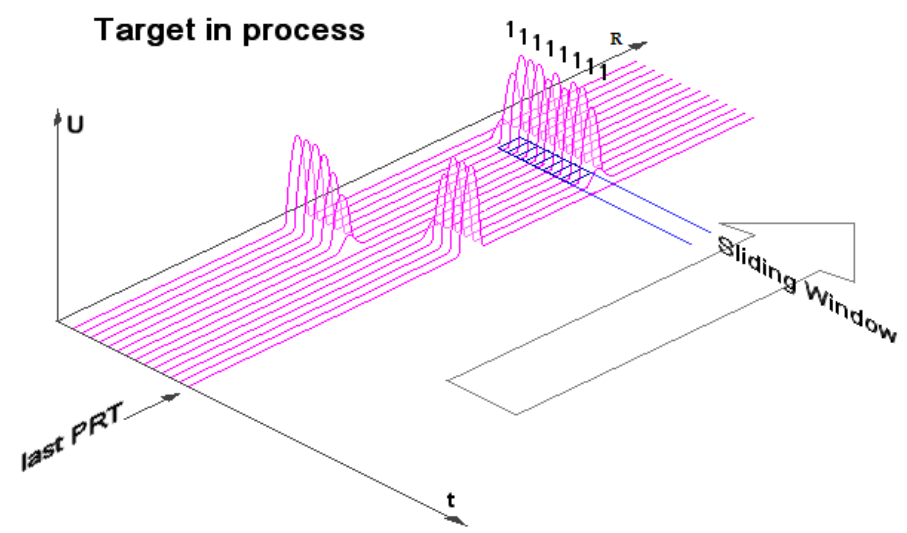

Figure 3. Moving window estimator process [5]

The moving window estimator has many disadvantages. For example, the estimated azimuth in (1) depends only on the first and last detected azimuths of the target. Therefore, the error of the estimated azimuth does not significantly decrease with increasing signal-to-noise ratio.

To avoid the disadvantages of the Moving window estimator, the "Beam shape centroid estimator" based on the collection of association and clustering algorithm was introduced for surveillance radar with coherent processing (see [7, 8]). However, both the Moving window and Beam shape centroid estimators give only the target azimuth estimate, it can not be applied for high range resolution radars, i.e. targets may spread over several adjacent range cells. For these cases, a more modern plot processor needs to be used for the correlation process than the simple sliding window.

One possible method is the Center of mass correlation [9]. The correlator is grouped first in range direction and then in azimuth direction (see Figures 4-6). The plot report is computed by using the center of mass of the signal processor output.

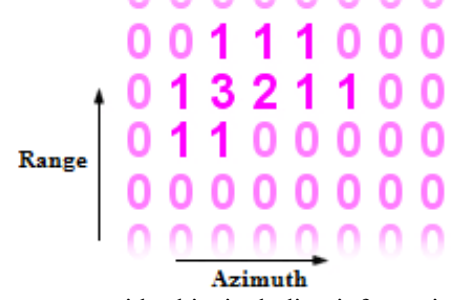

Figure 4. The Signal Processor provides hits including information about reveived power [9] 


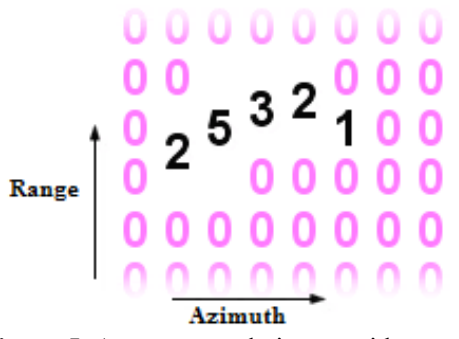

Figure 5. A range correlation provides groups [9]

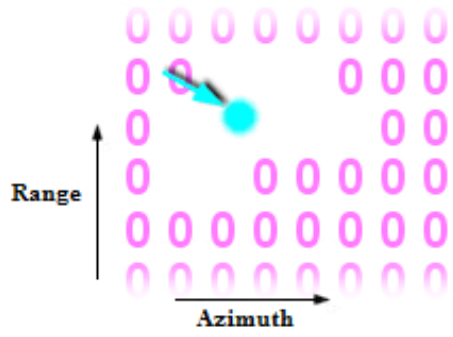

Figure 6. An azimuth correlation provides plots [9]

Some extensions of the Center of mass correlation can be found in [8-12].

For evaluation of the above mentioned methods researchers use the simulation data and calculate the range root-mean-square error (range RMSE) and bearing RMSE. However, the range-bearing centroid processing produces a series of time-dependent plot reports for each target (at every radar scan a plot for each target is reported). Therefore, the range and bearing RMSEs may not be enough to evaluate performance of the range-bearing centroid processing. Figure 7 presents two time series with the same RMSEs. But they have different behaviors. In fact, one of them (the dash-line) contains a shock (change-point), i.e. it is not structural stable.

So, in relation with the performance evaluation of range-bearing centriod processing there is a need to consider new parameters than RMSEs. In the next section, we propose the use of time series analysis for this purpose.

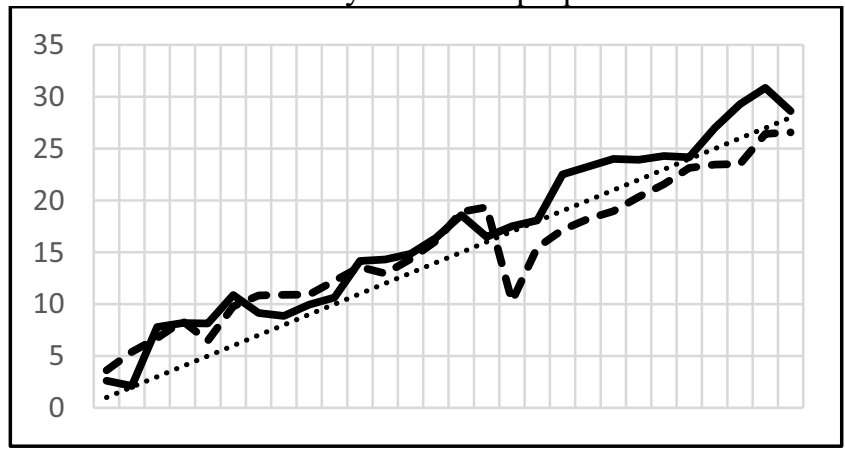

Figure 7. Two time series have the same RMSEs, but different behaviors. The dash-line series has a change-point. 


\section{Application of time series analysis for performance evaluation of range-bearing centroid processing}

Let $\{(R(k), B(k), P(k))\}$ be the range (in meter), bearing angle (in degree) and power (in Watt) of a target centroid (target plot report) at radar scan $k$. Three time series are available for analysis that are $\{(R(k))\},\{(B(k))\}$ and $\left\{\left(R^{4}(k) P(k)\right)\right\}$. The reason for considering the series $\left\{\left(R^{4}(k) P(k)\right)\right\}$ follows from the radar equation. It is well known (see [13], [14]) that the received signal power reflected from a target is determined by

where:

$$
P_{r}=\frac{P_{t} G_{t} G_{r} \sigma \lambda^{2}}{(4 \pi)^{3} R^{4}}
$$

- $\quad P_{r}$ is the received signal power $(\mathrm{W})$ at the radar receiver.

- $P_{t}$ is the radar transmitting power $(\mathrm{W})$.

- $G_{t}, G_{r}$ are the gain of the transmitting and receiving antennas, respectively.

- $\sigma$ is the radar cross section $\left(\mathrm{m}^{2}\right)$.

- $\quad \lambda$ is the wavelength $(\mathrm{m})$ of the radar electromagnetic energy.

- $\quad R$ is the target range $(\mathrm{m})$.

From (2) it follows that $P_{r} R^{4}$ is (theoretical) constant if the radar cross section $\sigma$ does not change. Two types of targets satisfied for the analysis are (see Figure 8):

- Type 1: targets that move along a straight line toward or away from the radar.

- Type 2: targets that move with a constant range to the radar.
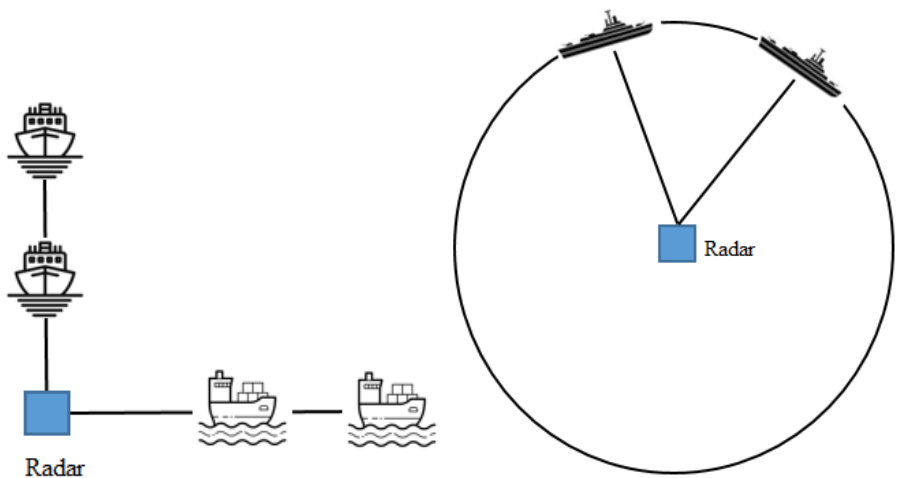

Figure 8. Illustration of targets of Type 1 (left) and of Type 2 (right)

The targets of type 1 and type 2 have (theoretical) constant radar cross section. Moreover, targets of type 1 do not change their bearing angles, so their series of bearing angles are useful for analysis while targets of type 2 do not change their ranges, so their series of ranges are used for evaluation.

We propose the new approach for the performance evaluation of the range-bearing centroid processing, which consists of

- accuracy evaluation using range RMSE and bearing RMSE;

- $\quad$ structural stability evaluation using time series analysis.

The Change-Point Ratio (CPR) is used for the evaluation of structural stability. Three values of CPR are estimated, that are bearing CPR (for a target of type 1), range CPR (for a target of type 2) and power CPR (for both types):

$$
\text { Range CPR }=\frac{\text { number of change }- \text { points of series }\{R(k)\}}{\text { Total number of points in series }\{R(k)\}}
$$




$$
\begin{gathered}
\text { Bearing CPR }=\frac{\text { number of change }- \text { points of series }\{B(k)\}}{\text { Total number of points in series }\{B(k)\}} \\
\text { Power } C P R=\frac{\text { number of change }- \text { points of series }\left\{P(k) R^{4}(k)\right\}}{\text { Total number of points in series }\left\{P(k) R^{4}(k)\right\}}
\end{gathered}
$$

The Matlab function findchangepts is applied for detection of change-points (see [15-17]). This function minimizes

$$
J(K)=\sum_{r=0}^{K} \sum_{i=k_{r}}^{k_{r+1}-1} \Delta\left(x_{i} ; \chi\left(\left[x_{k_{r}} \cdots x_{k_{r+1}-1}\right]\right)\right)+\beta K
$$

Where $K$ is the number of change-points; $k_{0}$ and $k_{K}$ are respectively the first and the last samples of the data; $\Delta$ and $\chi$ denote the deviation measurement and the emperical estimate which depend on the statistical property. For example,

- if 'Statitical property' is specified as 'mean':

$$
\Delta\left(x_{i} ; \chi\left(\left[x_{k_{r}} \cdots x_{k_{r+1}-1}\right]\right)\right)=\left(x_{i}-\operatorname{mean}\left(\left[x_{k_{r}} \cdots x_{k_{r+1}-1}\right]\right)\right)^{2}
$$

- if 'Statitical property' is specified as 'linear': Let the best-fit line through $x_{k_{r}}, \cdots, x_{k_{r+1}-1}$ be

$$
\hat{x}(t)=\frac{\left.S_{x t}\right|_{k_{r}} ^{k_{r+1}-1}}{\left.S_{t t}\right|_{k_{r}} ^{k_{r+1}-1}}\left(t-\operatorname{mean}\left(\left[t_{k_{r}} \cdots t_{k_{r+1}-1}\right]\right)\right)+\operatorname{mean}\left(\left[x_{k_{r}} \cdots x_{k_{r+1}-1}\right]\right)
$$

where

then

$$
\begin{gathered}
\left.S_{x y}\right|_{k_{r}} ^{k_{r+1}-1}=\sum_{k_{r}}^{k_{r+1}-1}\left(x_{i}-\bar{x}\right)\left(y_{i}-\bar{y}\right) \\
\bar{x}=\operatorname{mean}\left(\left[x_{k_{r}} \cdots x_{k_{r+1}-1}\right]\right) \\
\bar{y}=\operatorname{mean}\left(\left[y_{k_{r}} \cdots y_{k_{r+1}-1}\right]\right)
\end{gathered}
$$

$$
\Delta\left(x_{i} ; \chi\left(\left[x_{k_{r}} \cdots x_{k_{r+1}-1}\right]\right)\right)=\left(x_{i}-\hat{x}\left(t_{i}\right)\right)^{2}
$$

Test result. For the test we use the data from an X-band coastal surveillance radar in which the center of mass correlation is used for the range-bearing centroid processing. A target of type 1 (see Figure 9) is chosen for the evaluation of structural stability of centroid processing. In real scenario, we understand that type 1 contains all targets that move in a straight line with a small change of their bearing angles (not exceeding 5 degree). The bearing angles of the test target are in the interval from 110 degree to 115 degree. 


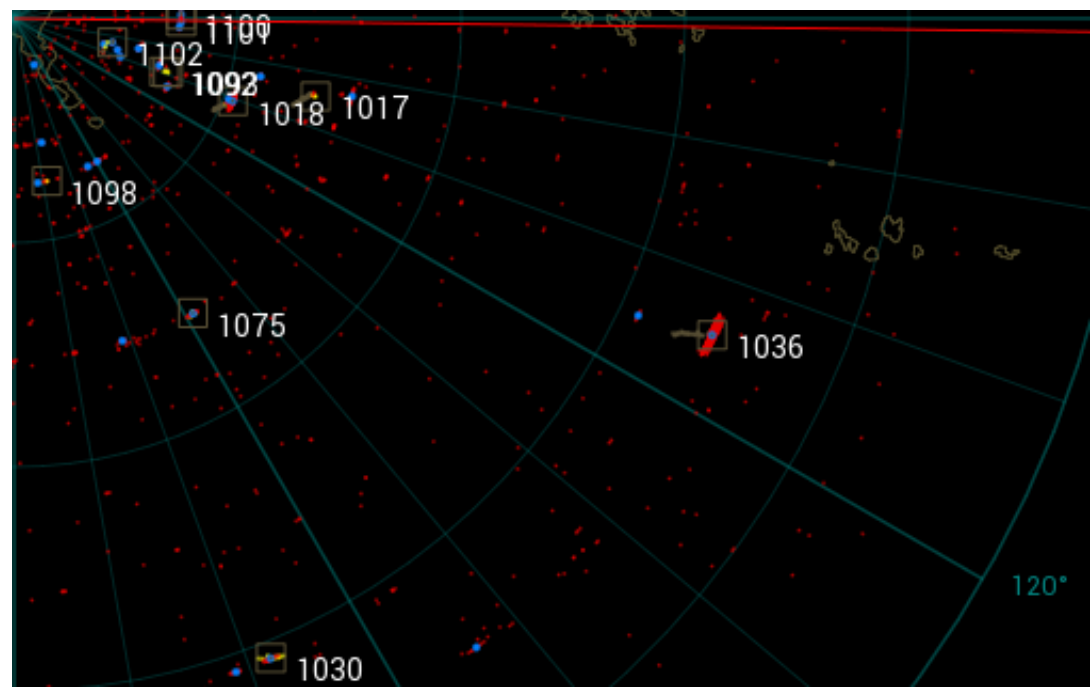

Figure 9. The test target (ID 1036) in the radar screen

Two data series $\{(B(k))\}$ and $\left\{\left(P(k) R^{4}(k)\right)\right\}$ are used for analysis of structural stability of range-bearing centroid processing. The parameters for the function findchangepts are set by:

- $\quad$ 'Statistic' = 'linear' (since the target moves in a straight line);

- 'MinThreshold' = 0 (to find all possible change-points);

- 'MinDistance' $=30$ (to reject the random noises in the change-points. The value 30 means that minimum number of samples between change-points is 30 , or equivalent to 5 minutes of radar scan data. Each radar scan is 10 seconds).

The test results are given in Figures 10 and 11.

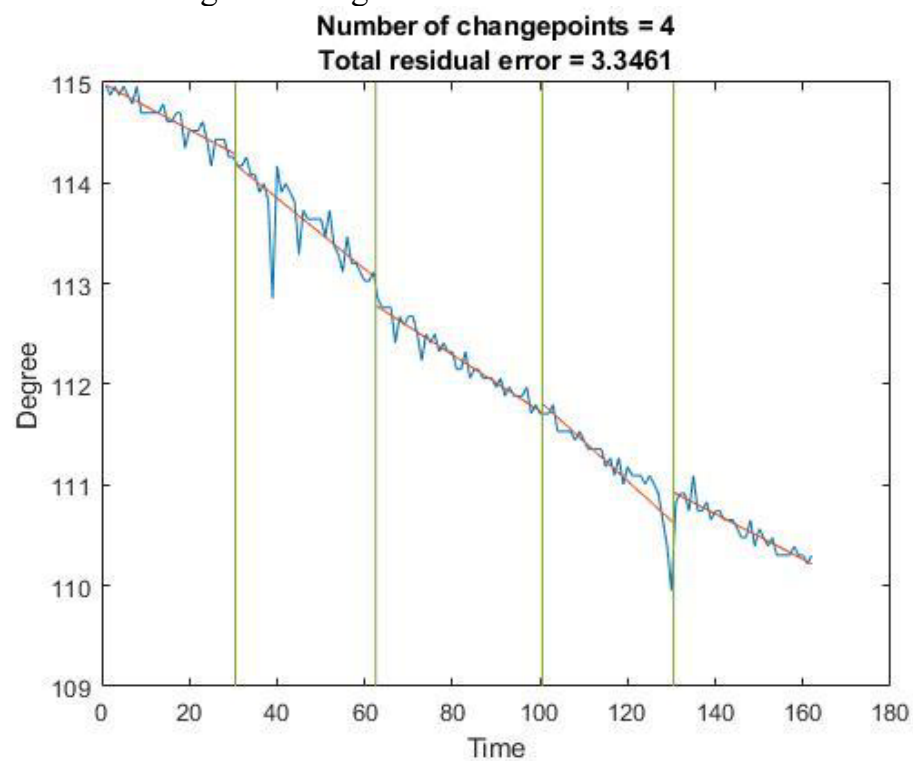

Figure 10. The change-points for the series $\{(B(k))\}$ of the test target. Bearing CPR $=4 / 162$. 


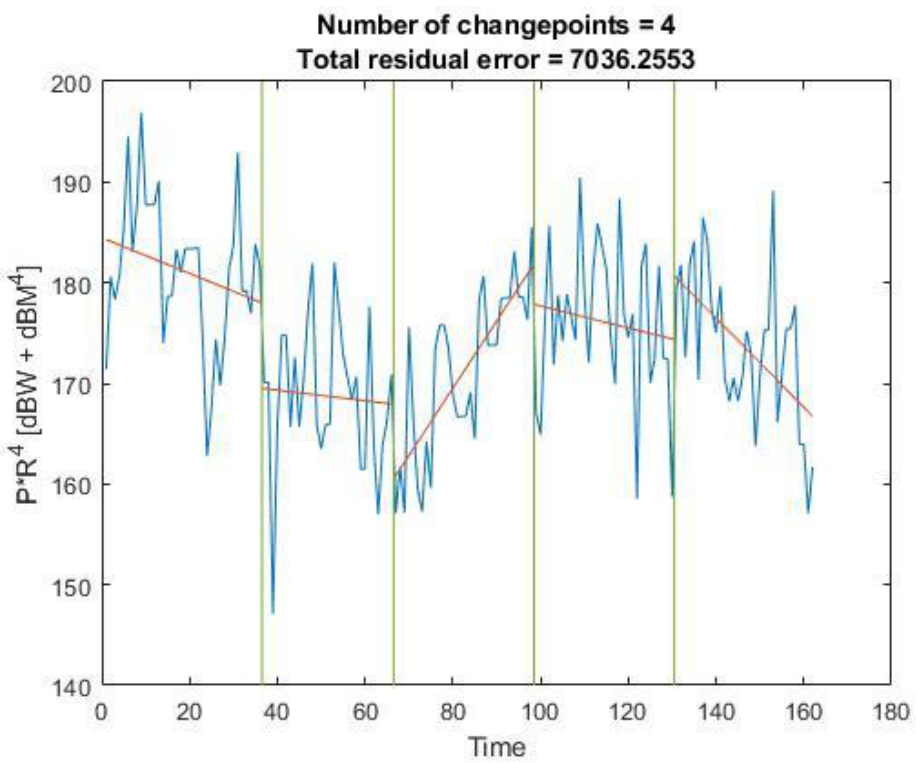

Figure 11. The change-points for the series $\left\{\left(P(k) R^{4}(k)\right)\right\}$ of the test target. Power $C P R=4 / 162$.

From Figures 10 and 11 it follows that the series $\{(B(k))\}$ and $\left\{\left(P(k) R^{4}(k)\right)\right\}$, and hence the range-bearing centroid processing, are not structural stable. The causes of the unstability of range-bearing centroid processing may occur in "Plot detector" or "Plot extractor" (see Figure 2). If the causes are in "Plot extractor" the radar designers need to use another centroid processing algorithm to avoid the structural unstability. For comparison, it is clear that the evaluation method used in [4]-[12] could not be able to detect the change-points while the proposed method gives us the detailed structural stability of the centroid processing. The unstability of the centroid processing will lead to a poor performance of "Tracking and Parameter estimation" block.

\section{Conclusion and Future works}

We have proposed a new (offline) approach for evaluation of range-bearing centroid processing for a radar system that consists of the accuracy evaluation using range and bearing RMSEs and the structural stability evaluation using time series analysis. A test with data from an X-band coastal surveillance radar is given for illustration.

In future works, we will study the online performance evaluation and its application for automatic calibration of a radar system. This will use the online time series analysis which is intensively investigated recently. 
Acknowledgement. The authors would like to thank the reviewers for their valuable comments and suggestions to improve the manuscript.

\section{References}

[1] https://en.wikipedia.org/wiki/Radar

[2] O'Donnell RM. Introduction to Radar Systems. Massachusetts Institute of Technology: MIT OpenCourseWare. 2007; http://ocw.mit.edu

[3] https://www.radartutorial.eu/10.processing/sp08.en.html

[4] Walker CM, Atkin J, Bickel H. Comparative evaluation of several azimuth estimating procedures using digital processing and search radar simulation. IRE Transactions on Aeronautical and Navigation Electronnics. 1958 Jun; p. 114-121.

[5] https://www.radartutorial.eu/10.processing/sp11.en.html

[6] Galati G, Struder FA. Angular accuracy of the binary moving window radar detector. IEEE Transactions on Aerospace and Electronic Systems; 1982 July; 18: 416-422.

[7] Cole EL, Hodges MJ, Oliver RG, Sullivan AC. Novel accuracy and resolution algorithms for the third generation MTD. Proc. of the 1986 National Radar Conference; 1986; p. 41-47.

[8] Slocumb BJ. Surveillance radar range-bearing centroid processing. Proc. SPIE 4473; Signal and Data Processing of Small Targets 2001; 2001 Nov. $26^{\text {th }}$.

[9] https://www.radartutorial.eu/10.processing/sp13.en.html

[10] Slocumb BJ, Macumber DL. Surveillance radar range-bearing centroid processing: Part II, Merged measurements. Proc. SPIE 6236; Signal and Data Processing of Small Targets 2006; 2006 Apr.

[11] Jian T, Xu X, Zhang L, Han Z. Two algorithms of plots centroid based on mass centre. J. of Air Force Radar Academy 2009; 23: 20-21.

[12] Liu HB, Li JB, Zhang P. A new algorithm of plots centroid for radar targets. Proc. of $9^{\text {th }}$ Int. Congress on Image and Signal Processing, BioMedical Engineering and Informatics; Datong, China; 2006 Oct. 15-17, p. 1268-1272.

[13] Skolnik MI (Edi. in Chief). Radar Handbook. McGraw-Hill; 2008; $3^{\text {rd }}$ ed.

[14] Barton DK. Radar Equation for Modern Radar. Artech House; 2013.

[15] Lavielle M. Using penalized contrasts for the change-point problem. Signal Processing 2005; 85: 15011510.

[16] Killick R, Fearnhead P, Ecklay IA. Optimal detection of change-points with a linear computational cost. J. of the Americal Statistical Association 2012; 107(500): 1590-1598.

[17] https://www.mathworks.com/help/signal/ref/findchangepts.html 